\title{
Lipoprotein receptors and a Disabled family cytoplasmic adaptor protein regulate EGL-17/FGF export in C. elegans
}

\author{
Darren M. Kamikura and Jonathan A. Cooper ${ }^{1}$ \\ Fred Hutchinson Cancer Research Center, Seattle, Washington 98109, USA
}

\begin{abstract}
Growth factors and morphogens need to be secreted to act on distant cells during development and in response to injury. Here, we report evidence that efficient export of a fibroblast growth factor (FGF), EGL-17, from the Caenorhabditis elegans developing vulva requires the lipoprotein receptor-related proteins Ce-LRP-1 and Ce-LRP-2 and a cytoplasmic adaptor protein, Ce-DAB-1 (Disabled). Lipoprotein receptors are transmembrane proteins best known for their roles in endocytosis. Ce-LRP-1 and Ce-LRP-2 possess a conserved intraluminal domain that can bind to EGL-17, as well as a cytosolic FXNPXY motif that can bind to Ce-DAB-1. Ce-DAB-1 contains signals that confer subcellular localization to Golgi-proximal vesicles. These results suggest a model in which Ce-DAB-1 coordinates selection of receptors and cargo, including EGL-17, for transport through the secretory pathway.
\end{abstract}

[Keywords: Selective secretion; fibroblast growth factor; lipoprotein receptor traffic; Dab; cargo selection; endocytosis]

Received March 24, 2003; revised version accepted September 22, 2003.

Many developmental morphogens and growth factors have specialized mechanisms of secretion. For example, Wingless $(\mathrm{Wg})$ secretion requires posttranslational $\mathrm{N}$-glycosylation by the transmembrane protein Porcupine (Porc) within the endoplasmic reticulum (van den Heuvel et al. 1993; Tanaka et al. 2002), whereas a Porc relative, Central missing (Cmn), is critical for Hedgehog (Hh) export (Amanai and Jiang 2001). Spitz, a transmembrane epidermal growth factor (EGF), is held within the ER until Star chaperones it to the Golgi apparatus, where it then encounters Rhomboid-1, which triggers proteolytic cleavage and allows Spitz to move to the cell surface (Lee et al. 2001). Most fibroblast growth factors (FGFs) act cell nonautonomously to coordinate cell migrations, proliferation, and fate specification during wound healing, angiogenesis, tumor metastasis, and development (Friesel and Maciag 1995; Goldfarb 1996; Sutherland et al. 1996; Borland et al. 2001). The majority of FGFs contain standard signal sequences for secretion by the ER-Golgi pathway. However, FGFs 1, 2, and 11-14 lack signal sequences, and FGFs 9, 16, and 20 have a hydrophobic $\mathrm{N}$ terminus that may not be cleaved (Friesel

${ }^{1}$ Corresponding author.

E-MAIL jcooper@fred.fhcrc.org; FAX (206) 667-6522.

Article and publication are at http://www.genesdev.org/cgi/doi/10.1101/ gad.1136103. and Maciag 1995; Ornitz and Itoh 2001). Thus, certain FGFs may use distinct mechanisms for secretion.

A Caenorhabditis elegans fibroblast growth factor (FGF), EGL-17 (egg-laying defective), regulates the migrations of the sex myoblasts (SMs) during larval development (DeVore et al. 1995; Burdine et al. 1997, 1998). The SMs are born at the posterior of L1 stage larvae and migrate anteriorly to the gonad center, where they generate the uterine and vulval musculature (Thomas et al. 1990). EGL-17 is expressed in the developing vulva and gonad, and acts as an attractive cue for the SMs, which express an FRF receptor (FGFR), EGL-15. Mutations in genes for either the ligand (egl-17) or receptor (egl-15) prevent correct SM positioning around the gonad center, and egglaying defects result (Stern and Horvitz 1991). Although these data indicate that EGL-17 is secreted, the mechanism by which this occurs has not been investigated.

Here we report that efficient export of EGL-17 by the developing vulva involves the adaptor protein Disabled (Ce-DAB-1) and two lipoprotein receptor-related proteins, Ce-LRP-1 and Ce-LRP-2. Ce-DAB-1 and the receptors appear to function posttranscriptionally to promote secretion. Lipoprotein receptors cycle rapidly through the endocytic pathway, bringing bound ligands into the cell and releasing them in the acidic environment of endosomes before returning to the cell surface (Nykjaer and Willnow 2002). Their extracellular domains contain variable numbers of repeated domains that allow them 
to interact with a diverse set of macromolecules bearing little structural similarity (Herz and Strickland 2001; Christensen and Birn 2002). Although the primary roles of lipoprotein receptors appear to be in endocytosis, recent evidence suggests that they may participate in the transcytosis of at least some of their ligands (Dehouck et al. 1997; Marino et al. 2000, 2001). However, there is no evidence regarding a possible role in export of proteins from the cell. Ce-DAB-1 is related to murine Dab1, which functions in a signaling pathway together with lipoprotein receptors VLDLR (very low density lipoprotein receptor) and ApoER2 (Apolipoprotein E receptor), and Dab2, which regulates endocytosis of lipoprotein receptors Megalin and LDLR (Howell and Herz 2001; Mishra et al. 2002a; Morris et al. 2002). Dab1 and Dab2 contain a PTB (phosphotyrosine-binding) domain, also found in Ce-DAB-1, which mediates their association with an FXNPXY motif that is conserved in the intracellular domains of lipoprotein receptors (Howell et al. 1999; Trommsdorff et al. 1999; Morris and Cooper 2001). The FXNPXY motif in the LDL (low-density lipoprotein) receptor is critical for endocytosis and also for trafficking to the basolateral surface of polarized epithelial cells (Nelson and Yeaman 2001). The binding of Dab proteins to a conserved signal on lipoprotein receptors suggests that they may directly regulate receptor traffic.

\section{Results}

\section{Roles of Ce-dab-1 during larval development}

The C. elegans gene Ce-dab-1 encodes a protein related to Disabled (Dab) family members in mice and Drosophila (Fig. 1A). The gene appears to be alternatively spliced, but all predicted proteins contain a conserved PTB domain and, like mammalian Dab2, have several sequences that may confer binding to clathrin adaptor proteins (Fig. 1A, red triangles) and Eps15 homology (EH) domain proteins (blue circles).

To identify a role for $C e$-dab-1, gene function was disrupted by using RNA interference (RNAi; Fire et al. 1998). Stage L1 larvae were soaked in Ce-dab-1 or control double-stranded RNA (dsRNA; Tabara et al. 1998; Maeda et al. 2001). A number of phenotypes were reproducibly observed in the $\mathrm{F}_{\mathrm{O}}$ generation, including highly penetrant defects in cuticle molting (Mlt) and in egg laying ( $\mathrm{Egl}$; Table 1). dsRNA prepared from alternate regions of Ce-dab-1 conferred the same phenotypes, indicating specificity (Table 1).

The Mlt defect of Ce-dab-1 RNAi-treated worms resembled that caused by mutation of Ce-lrp-1, which encodes a Megalin relative (Yochem et al. 1999). The cuticle formed blisters, and some worms dragged cuticle remnants behind them. Many adults had a "wasp waist"
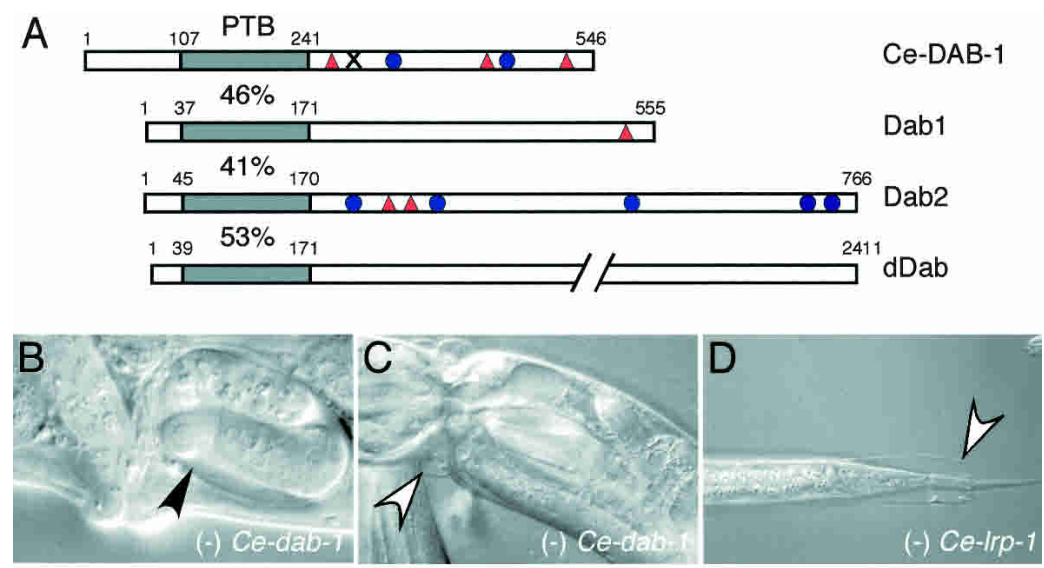

E

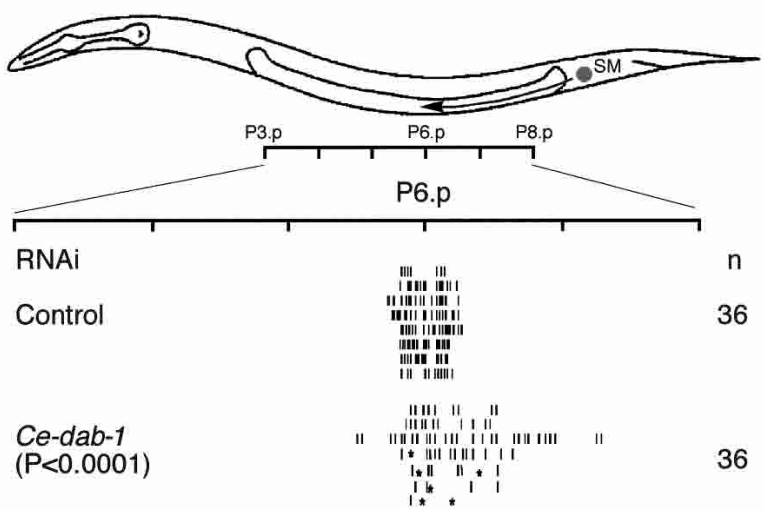

Figure 1. Characterization of $C e-D A B-1$ function and expression. $(A)$ Structural schematic of Ce-DAB-1 protein compared with mouse Dab1 and Dab2 and Drosophila Dab. Blue circles represent NPF sequences and potential binding sites for $\mathrm{EH}$ domain proteins. Red triangles represent DPF sequences and potential binding sites for clathrin adaptors. An " $\mathrm{X}$ " labels the approximate boundary between $5^{\prime}$ and $3^{\prime}$ portions of the cDNA used to generate RNAi. Percentage of identity between the PTB domains relative to Ce-DAB- 1 (shaded) is indicated. $(B-D)$ RNAi phenotypes. $C e-d a b-1$ (RNAi) animals exhibit deficiencies in egg laying as adults $(B)$. Black arrowhead indicates an embryo at threefold stage $(\sim 525$ cells). Ce-dab-1 (RNAi) animals are also deficient in cuticle molting $(C)$ and resemble lrp-1 RNAi animals $(D)$, exhibiting blisters, "wasp-waist," and girdle of unshed cuticle (white arrowhead). (E) Ce-dab-1 (RNAi) disrupts SM migration. The circle labeled "SM" marks the approximate position at which the SMs are generated. The nuclei of the Pn.p cells, a set of hypodermal cells along the ventral side of the animal, are used as an anatomical ruler for the measurement of the final positions of the SMs. A vertical hash mark indicates the final position of an individual SM, with an asterisk $\left({ }^{*}\right)$ indicating an individual dorsally localized SM. RNAi used (control or Ce$d a b-1)$ is indicated in the left column, and the number of worms scored is indicated in the right column. Statistical significance of altered SM distribution in Ce-dab-1 versus control (RNAi) animals is indicated. 
Table 1. Ce-dab-1 RNAi phenotypes

\begin{tabular}{lccccc}
\hline RNAi $^{\mathrm{a}}$ & $\%$ egl $^{\mathrm{b}}$ & Laying rate $(\mathrm{eggs} / \mathrm{h})^{\mathrm{c}}$ & Stage $^{\mathrm{d}}$ & \% mlt $^{\mathrm{e}}$ & Animals scored $^{\mathrm{f}}$ \\
\hline Control & $1.5 \pm 1.4$ & $4.8 \pm 1.2$ & $\sim$ gastrulation & 0 & 720 \\
Ce- dab-1 & & & & & \\
$1-546^{\mathrm{g}}$ & $74.9 \pm 9.7$ & $1.8 \pm 0.9$ & $\sim$ pretzel & $26.1 \pm 6.3$ & 249 \\
$1-293$ & $77.7 \pm 5.7$ & $\mathrm{nd}$ & $\sim$ pretzel & $25.6 \pm 14.6$ & 257 \\
$290-546$ & $75.9 \pm 5.2$ & $\mathrm{nd}$ & $\sim$ pretzel & $31.8 \pm 10.2$ & 161 \\
\hline
\end{tabular}

${ }^{\mathrm{a}} \mathrm{L} 1$ animals were soaked overnight in solutions of dsRNA and scored as young adults. Control RNAi was prepared from an empty vector.

${ }^{\mathrm{b}}$ Egg-laying defective animals were scored as young adults by using DIC microscopy and looking for postgastrulation embryos.

${ }^{\mathrm{c}}$ The number of eggs laid by individual animals was counted over several hours and averaged $(\mathrm{n}=10)$.

${ }^{\mathrm{d}}$ The approximate stage of eggs upon laying were marked as either $\sim$ gastrulation stage (30 to 150 cells) or $\sim$ pretzel stage (400 to 550 cells).

${ }^{\mathrm{e}}$ Molting-deficient animals were scored by DIC as predominantly young adults. Only animals with body constrictions due to unshed cuticle were scored as positive.

${ }^{\mathrm{f}} \mathrm{Numbers}$ represent a total of six independent experiments.

${ }^{\mathrm{g}} \mathrm{Numbers}$ represent residues in the corresponding protein sequence.

${ }^{\mathrm{h}} \mathrm{nd}$ indicates not determined.

close to the vulva, apparently due to a girdle of unshed cuticle (Fig. 1C). The same phenotype was induced by Ce-lrp-1 RNAi (Fig. 1D) or cholesterol starvation (Yochem et al. 1999). This suggests that Ce-dab-1 and Celrp-1 may regulate cholesterol availability for cuticle synthesis or molting.

Ce-dab-1 RNAi worms laid eggs at a slower rate and later stage than did control animals (Fig. 1B; Table 1). Egl phenotypes can result from deficiencies in the development of the vulva, gonad, or egg-laying muscles (Sulston and Horvitz 1977; Newman et al. 1996). The vulvae and gonads of Ce-dab-1 (RNAi) animals appeared to be normal, and the egg-laying rate did not correlate with the presence of cuticle fragments in or near the vulva, suggesting that the Egl phenotype was not caused by physical blockage. We therefore examined the egg-laying muscles, using worms expressing green fluorescent protein (GFP) in their SMs (Harfe et al. 1998). Depletion of Ce-dab-1 caused significant posterior displacement of the SMs $(P \leq 0.0001$; Fig. 1E). These defects were similar to, but less severe than, those caused by egl-17 mutation (Burdine et al. 1997, 1998). This suggests that Ce-dab-1 may be needed for proper functioning of the EGL-17/ EGL-15 signaling pathway.

\section{Ce-dab-1 is expressed in the developing vulva}

To distinguish whether Ce-DAB-1 regulates SM migration through the EGL-17 producing cells or the EGL-17 responding SMs, we identified the sites of $\mathrm{Ce}$-dab-1 gene expression. We used $5.5 \mathrm{~kb}$ of $C e-d a b-1$ upstream sequence to express either a DAB-1::GFP translational fusion (Ce-dab-1::Ce-DAB-1::GFP; Fig. 2A) or GFP (Cedab-1::GFP; data not shown) in transgenic worms. L2 stage worms showed GFP fluorescence in the ventral hypodermis (Fig. 2A). In normal hermaphrodites, three hypodermal cells (the VPCs, P5.p, P6.p, and P7.p), give rise to the vulva (Fig. 2B; Sulston and Horvitz 1977). Expression of Ce-dab-1 within the VPCs and their descendants continued through vulval development, and became re- stricted to the descendants of P5.p and P7.p by mid-L4 (Fig. 2). Ce-dab-1 was also expressed in the anchor cell (AC), sheath cells surrounding the amphid neurons in the head, the gut, and several unidentified cells in the anus and uterus of L3-adult animals. However, no $\mathrm{Ce}$ dab-1 expression was detectable in the SMs. The expression of $C e-d a b-1$ in the developing vulva and gonad partially overlaps that reported for egl-17 (Fig. 2H; Burdine et al. 1998; Branda and Stern 2000), suggesting that Cedab-1 may regulate SM migration through an interaction with egl-17.

\section{Ce-DAB-1 prevents EGL-17 protein accumulation in the VPCs}

We considered that Ce-dab-1 might regulate egl-17 gene expression or EGL-17 protein secretion. Because of weak expression of EGL-17 reporter constructs in the developing gonad (Branda and Stern 2000), we monitored egl-17 expression in the descendants of P6.p. To test whether Ce-dab-1 regulates egl-17 promoter activity, we used animals expressing GFP from the egl-17 promoter (Fig. 3A; Burdine et al. 1998). Ce-dab-1 RNAi has no effect on the levels of egl-17::GFP expression during early (Fig. $3 \mathrm{~A}$ ) or late (data not shown) vulval development. Therefore, we tested whether Ce-dab-1 might regulate the export of EGL-17 from the developing vulva, by using the egl-17 promoter to express a translational fusion of fulllength EGL-17 to GFP (Fig. 3C). To determine whether the transgene is functional, it was injected into egl17(n1377) mutant animals. Animals bearing the transgene showed a pronounced reduction in the characteristic egl-17(n1377) "bag of worms" Egl phenotype (Fig. 3B). This suggests that the EGL-17::GFP fusion protein is exported and functional.

EGL-17:: GFP protein traffic was monitored by both live GFP and indirect immunofluorescence in wild-type egl-17::EGL-17::GFP larvae. Long exposures revealed weak punctate fluorescence in the daughters or granddaughters of P6.p (Fig. 3C, top). The rescue of the egl-17 

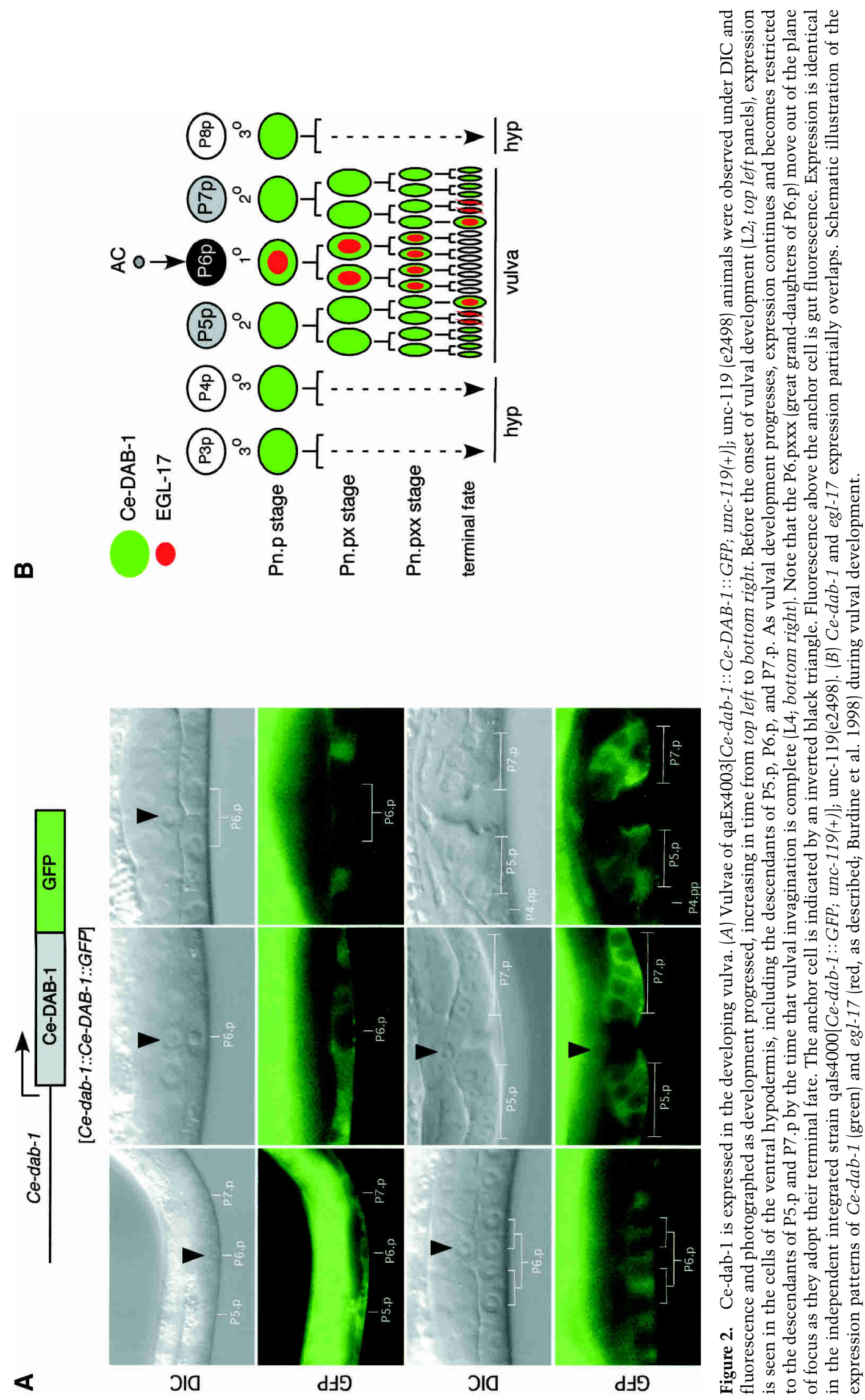


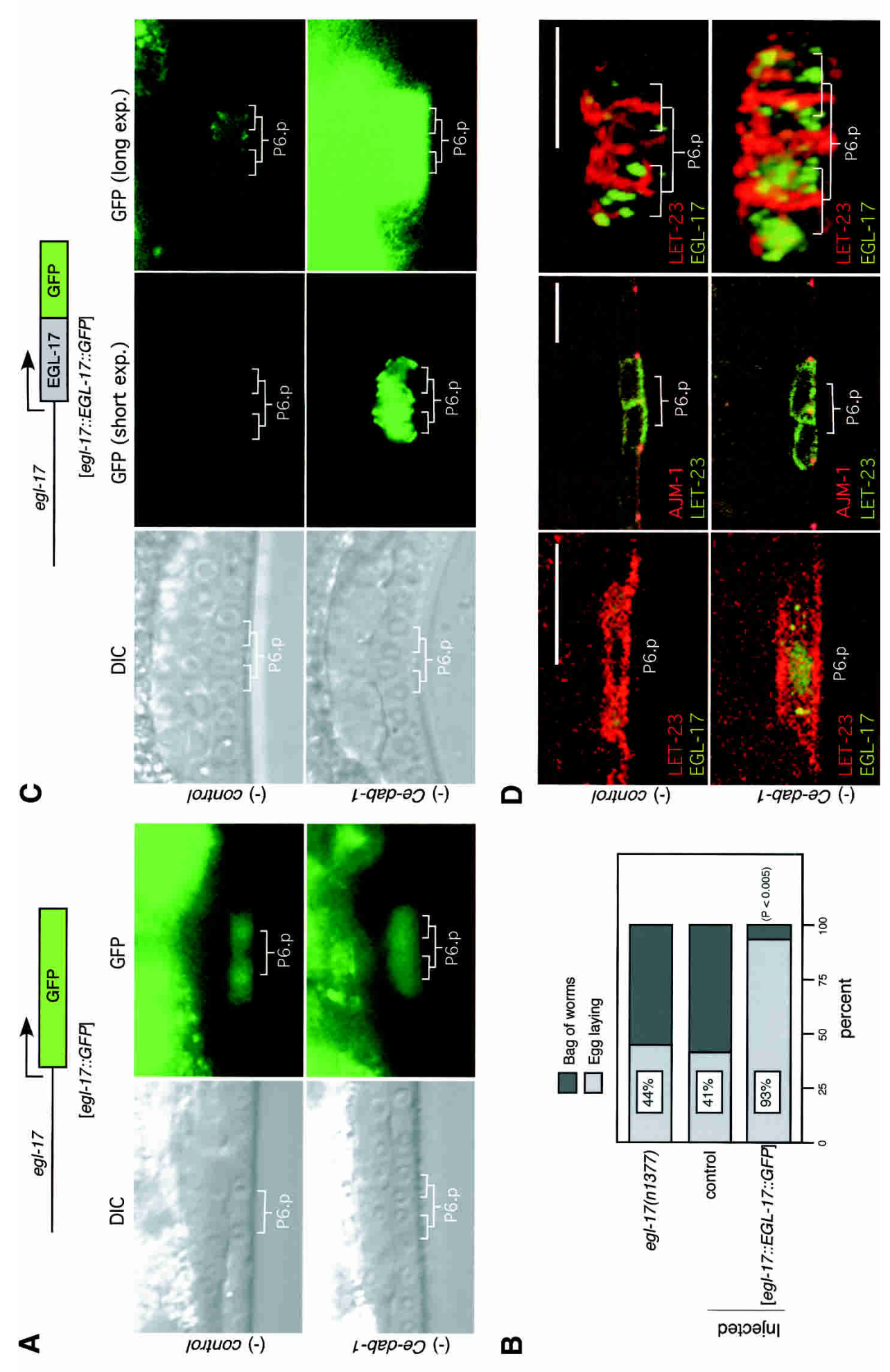

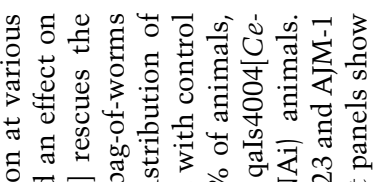

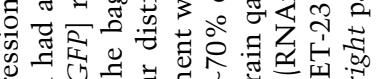

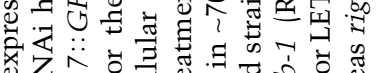

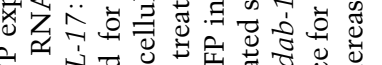

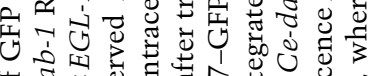
뭉 원.

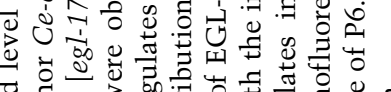

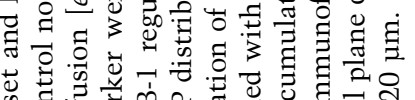

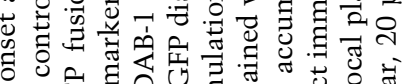

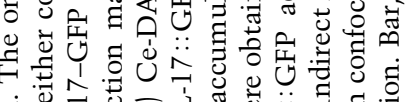

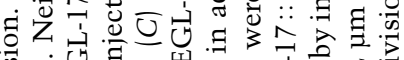
क⿻

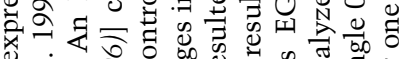

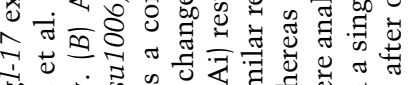
के

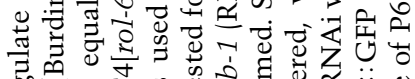

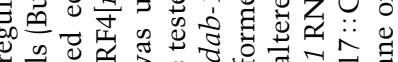

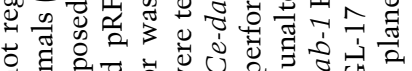

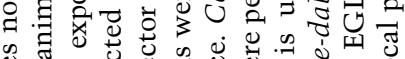
这

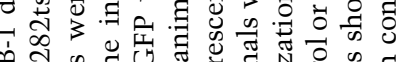

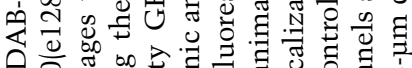

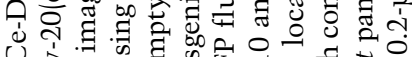

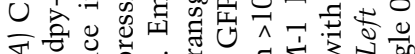

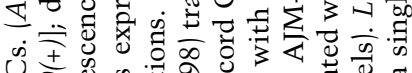

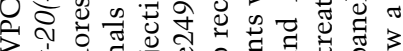

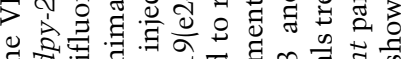

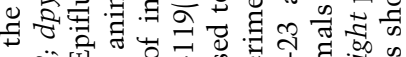

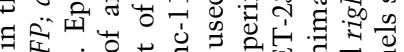

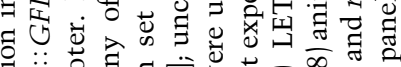

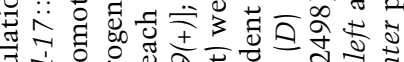

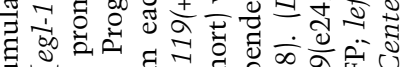
웡

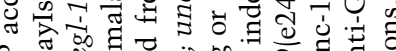

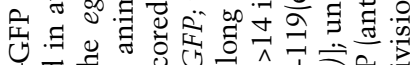

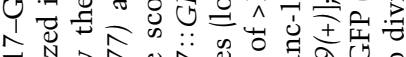

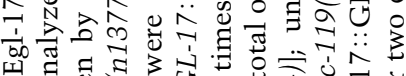
o 碀

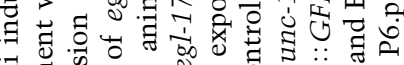

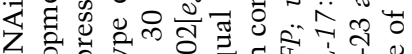

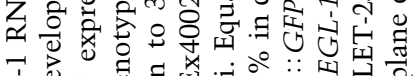

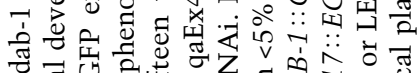
U。

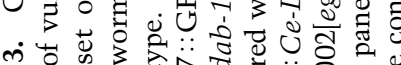
के

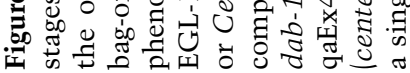


mutant phenotype and the pattern of fluorescence suggest that EGL-17::GFP is exported via vesicular structures from P6.p and its descendants and regulates SM migration. Ce-dab-1 RNAi introduced by either feeding (data not shown) or soaking caused a pronounced increase in fluorescence intensity in the descendants of P6.p (Fig. 3C, bottom). Indirect immunofluorescence shows an increased number of EGL-17::GFP-containing structures within P6.p and its descendants (Fig. 3D). Combined with the Egl and SM migration effects of $\mathrm{Ce}$ $d a b-1$ RNAi, this suggests that Ce-dab-1 RNAi inhibits EGL-17 secretion and results in its accumulation in the secretory pathway. The effect was specific, because the cell surface protein LET-23 and the junctional protein AJM-1 were localized normally in these cells despite the retention of EGL-17:: GFP (Fig. 3D).

To investigate whether accumulation of EGL-17::GFP can result from reduced secretion, we inhibited several genes implicated in vesicle traffic. The clathrin adaptor protein complex AP-1B is involved in basolateral secretion in mammalian cells (Folsch et al. 1999), whereas AP-3 is involved in Golgi/lysosome traffic and AP-2 in endocytosis (Lippincott-Schwartz et al. 2000). RNAi for $\mu 1 / \mu 1 \mathrm{~B}$ subunits of AP-1B (unc-101, apm-1, and apt-10; Shim et al. 2000) each caused pronounced EGL-17::GFP accumulation in the P6.p descendants but did not cause a Mlt defect (Table 2). RNAi for a $\sigma 1$ subunit of AP-1 (apt-2) caused both EGL-17::GFP accumulation and defective molting. Conversely, RNAi for potential $\delta$ and $\beta 3$ subunits of AP-3 (apt-5 and apt-6, respectively), had no phenotype, and RNAi for the $\alpha$-adaptin subunit of AP-2 (apt-4) was lethal. These results support the hypothesis that retention of EGL-17::GFP can be caused by impaired secretion, and also show that EGL-17::GFP retention is independent of cuticle molting. In addition, Ce$d a b-1$ is unlikely to have an essential role in AP-2 function.

Lipoprotein receptors Ce-LRP-1 and Ce-LRP-2 participate in EGL-17 export from the P6.p descendants

The sequence of Ce-DAB-1 indicates that it is cytoplasmic. Ce-DAB-1 might therefore regulate secretion by binding to transmembrane receptors present in vesicles that carry EGL-17. Lipoprotein receptors span the membrane and associate with Dab family proteins in vertebrates and, thus, might connect Ce-DAB-1 with EGL-17 in the C. elegans developing vulva.

C. elegans has at least three genes related to mammalian lipoprotein receptors: Ce-lrp-1, rme-2 (receptor-mediated endocytosis; Grant and Hirsh 1999), and a gene related to mammalian Lrp (named here Ce-lrp-2) contained in the cosmids F48B3 and T21E3 (Springer 1998). Ce-lrp-1 and Ce-lrp-2 expression has been detected in the developing vulva by in situ hybridization (Shin-i and Kohara 2001). Therefore, we used RNAi to test whether these genes are involved in SM migration. Although inhibition of any of the genes individually had no effect, combined RNAi of Ce-lrp-1 and Ce-lrp-2 caused a significant reduction in SM migration ( $P<0.0002$; Fig. 4A). $C e-d a b-1$ RNAi in addition to Ce-lrp-1 and Ce-lrp-2 had no additional effect on SM migration, suggesting that these genes may work in a common pathway. Rme-2 RNAi had no effect on SM migration when tested alone or in combination with Ce-lrp-1 or Ce-lrp-2 (Fig. 4A), suggesting that the effects of Ce-lrp-1 and Ce-lrp-2 RNAi are specific.

To test the roles of Ce-LRP-1 and Ce-LRP-2 in EGL-17 export, we observed their effects on egl-17 expression and EGL-17::GFP accumulation in P6.p descendants. Combined RNAi for Ce-lrp-1 and Ce-lrp-2 had no effect on egl-17 promoter activity (Fig. 4B) but did cause accumulation of EGL-17::GFP in these cells (Fig. 4C). As expected, RNAi for Ce-lrp-1 or Ce-lrp-2 individually had no effect in this assay (data not shown). These results suggest that Ce-LRP-1 and Ce-LRP-2 function with CeDAB-1 to facilitate EGL-17 export from P6.p descendants.

Ce-DAB-1 interacts with Ce-LRP-1 and Ce-LRP-2 and localizes to post-Golgi vesicles

In mammals, the PTB domains of Dab1 and Dab2 bind to a consensus FXNPXY in the intracellular domains of lipoprotein receptors (Howell et al. 1999; Morris and Cooper 2001). Ce-LRP-1 and Ce-LRP-2 contain such motifs. Therefore, we tested for Ce-DAB-1 PTB domain binding

Table 2. Adaptin complexes and EGL-17 secretion

\begin{tabular}{|c|c|c|c|c|c|}
\hline Gene $^{a}$ & Adaptin complex ${ }^{\mathrm{b}}$ & Subunit ${ }^{\mathrm{b}}$ & Function $^{\mathrm{b}}$ & EGL-17::GFP accumulation ${ }^{\mathrm{c}}$ & $\mathrm{mlt}^{\mathrm{d}}$ \\
\hline apm-1 & AP-1 & $\mu 1 / \mu 1 \mathrm{~B}$ & secretion & + & - \\
\hline apt-10 & & & & + & - \\
\hline unc-101 & & & & + & - \\
\hline apt-2 & & & & + & + \\
\hline apt-4 & AP-2 & $\alpha$ & endocytosis & $\mathrm{n} / \mathrm{a}^{\mathrm{e}}$ & $\mathrm{n} / \mathrm{a}^{\mathrm{e}}$ \\
\hline apt-5 & AP-3 & $\delta$ & golgi-lysosome traffic & - & - \\
\hline apt-6 & & $\beta 3$ & & - & - \\
\hline
\end{tabular}

${ }^{a}$ L1 animals were soaked overnight in solutions of dsRNA and scored as L3 larvae. Control RNAi was prepared from an empty vector.

bPesumptive adaptin complex, subunit, and function by homology with mammalian proteins.

${ }^{\mathrm{c}}$ Accumulation of the EGL-17::GFP fusion protein was measured in the descendants of P6.p as described.

${ }^{\mathrm{d}}$ Molting-deficient animals were scored by DIC as L3/L4 larvae.

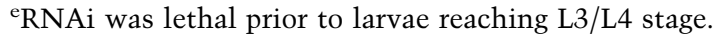


A

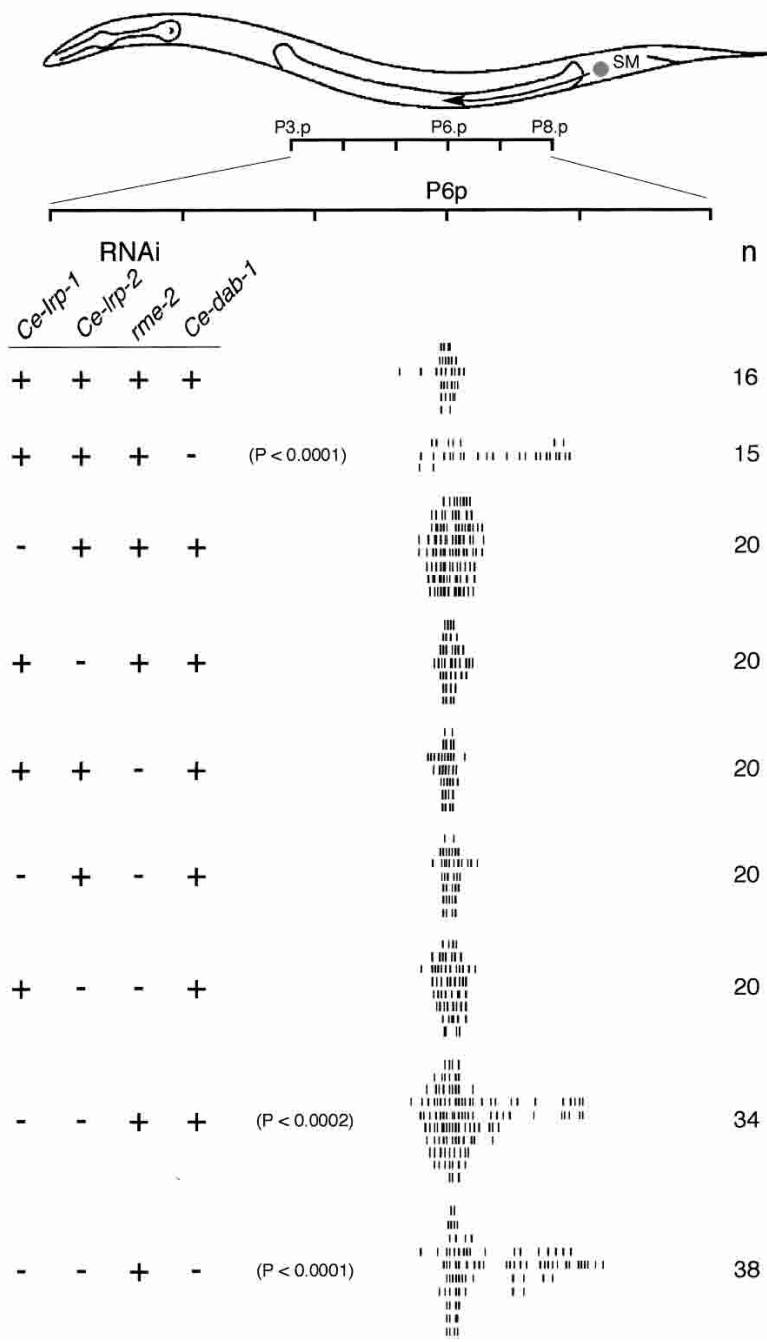

B

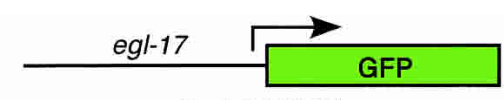

$[e g l-17:: G F P]$

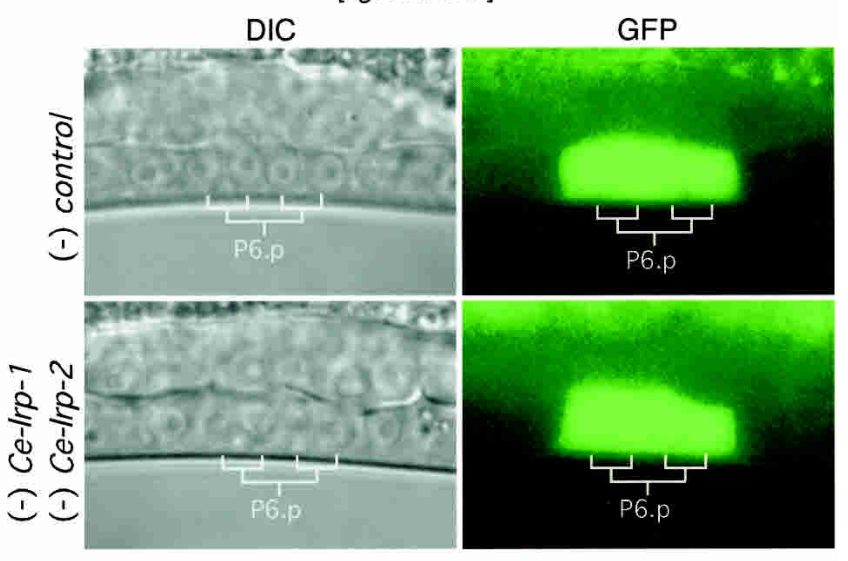

C

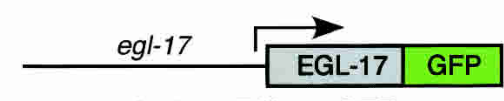

$[e g l-17:: E G L-17:: G F P]$

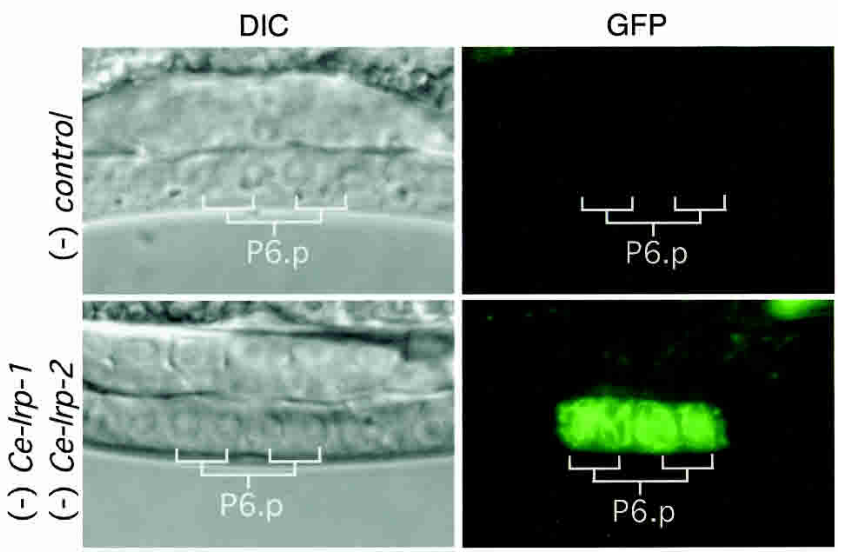

Figure 4. Lipoprotein receptors Ce-LRP-1 and Ce-LRP-2, but not RME-2, regulate SM migration. (A) Ce-LRP-1 and Ce-LRP-2 regulate SM migration. The positions of individual SMs were measured as in Figure 1. dsRNA was added as indicated: $(-)$ RNAi present; $(+)$ no RNAi. Numbers in the right column indicate the number of animals scored. (B) The lipoprotein receptors Ce-LRP-1 and Ce-LRP-2 do not regulate EGL-17 expression. egl-17::GFP animals were analyzed after treatment with control or Ce-1rp-1; Ce-lrp-2 RNAi. (C) Ce-LRP-1 and Ce-LRP-2 regulate EGL-17 export from the VPCs. qaEx4002[egl-17::EGL-17::GFP; unc-119(+)]; unc-119(e2498) transgenic animals were tested for changes in subcellular distribution of the EGL-17::GFP fusion after treatment with control or Ce-lrp-1; Ce-lrp-2 RNAi.

to the intracellular domains of Ce-LRP-1 and Ce-LRP-2. Using the yeast two-hybrid system, both Ce-LRP-1 and Ce-LRP-2 interact with Ce-DAB-1 (Fig. 5A,B). Ce-DAB-1 PTB domain binding to Ce-LRP-1 was reduced by mutation of the consensus FXNPXY motif from amino acids 4653-4658 but not by mutation of related motifs (Fig. $5 \mathrm{C}, \mathrm{D})$, suggesting evolutionary conservation of the Dab PTB binding site. Ce-DAB-1 also contains sequence motifs that may permit binding to clathrin adaptor complexes (Fig. 1A), suggesting that it might localize to coated vesicles. Because there are currently few markers for subcellular compartments in C. elegans VPCs, we observed the subcellular localization of Ce-DAB-1 in mammalian tissue culture cells. As shown in Figure 5E,
Ce-DAB-1 localized to vesicular structures, including AP-1-containing, wheat germ agglutinin-positive structures near the Golgi. Ce-DAB-1 did not localize with late endosomes (Lamp-1 marker) and localized poorly with concanavalin A-positive membranes. In addition, by indirect immunofluorescence, Ce-DAB-1 is also localized to vesicular structures in the P6.p descendants of animals expressing a Ce-DAB-1::GFP fusion protein (Fig. $5 \mathrm{~F})$. This localization suggests that Ce-DAB-1 may regulate traffic of vesicles carrying Ce-LRP-1 or Ce-LRP-2 by interacting with other proteins involved in coating or targeting post-Golgi traffic. However, identification of the step in the EGL-17 secretory pathway regulated by Ce-DAB-1 will require additional experiments. 
A

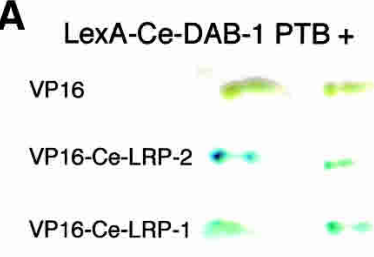

B

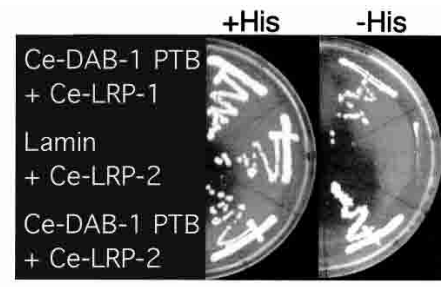

C Ce-LRP-1

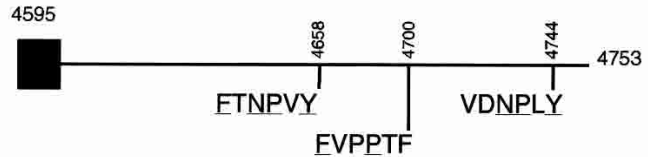

D LexA-Ce-DAB-1 PTB +

E
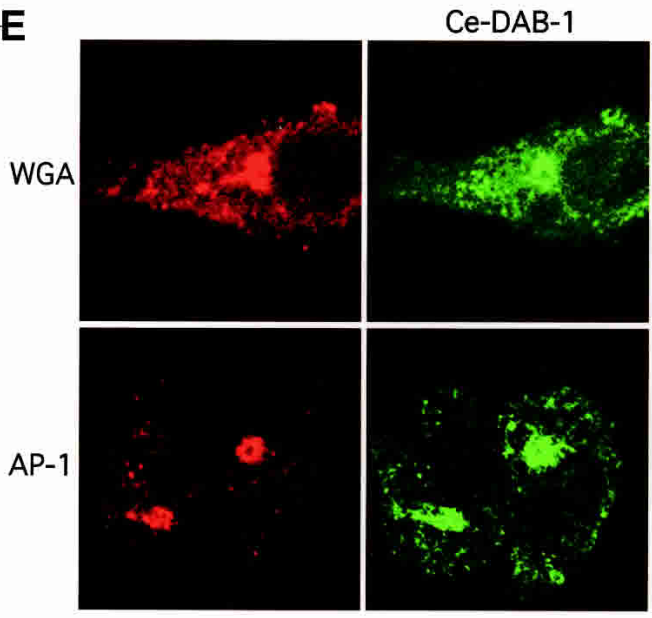

$\mathbf{F}$

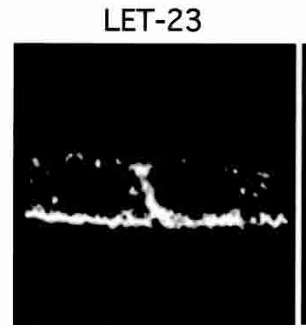

Ce-DAB-1:GFP

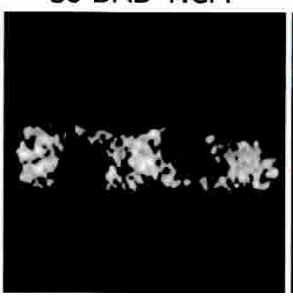

+ His
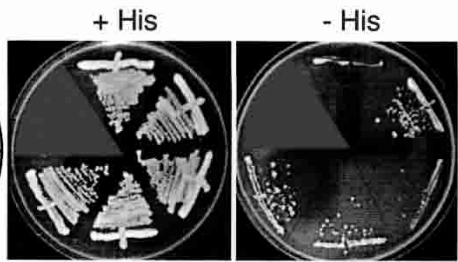

merge
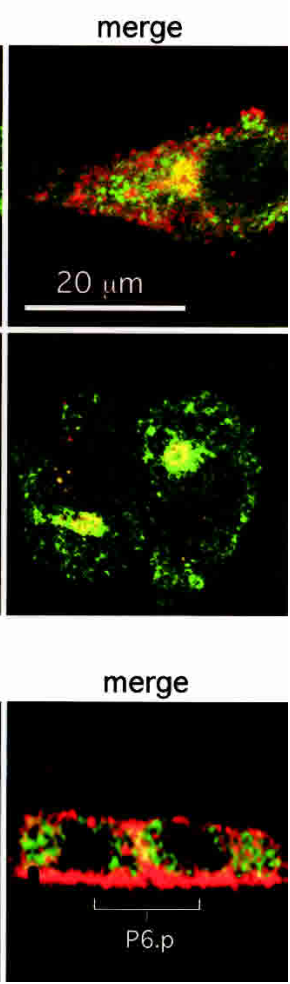

Figure 5. Ce-DAB-1 associates with Ce-LRP-1 and CeLRP-2 and with Golgi components. $(A, B)$ The CeDAB-1 PTB domain (residues 1-252) shows interaction with the intracellular domains of both Ce-LRP-1 (residues 4595-4753) and Ce-LRP-2 (C-terminal 81 residues) by yeast two-hybrid assay. $\beta$-Galactosidase activity (replicates; $A)$ and growth in the absence of histidine $(B) .(C)$ Potential binding sites in Ce-LRP-1 (underlined amino acids indicate those that match the consensus binding site for the PTB domains of mammalian Dab1 and Dab2). (D) The Ce-DAB-1 PTB domain interacts with an FXNPXY motif from residues 4653-4658. Substitution of Tyr 4658 with alanine reduces interaction between the Ce-DAB-1 PTB domain and the intracellular domain of Ce-LRP-1. (E) Subcellular localization of Ce-DAB-1 expressed in tissue culture cells. NIH3T3 (WGA) and HeLa cells ( $\gamma$-adaptin) were transfected to express Ce-DAB-1 and processed for indirect immunofluorescence with antibodies to $\gamma$-adaptin subunit AP- 1 , Ce-DAB-1, and directly labeled WGA. Note that there are two HeLa cells in the image shown. $(F)$ Ce-DAB$1:: \mathrm{GFP}$ is present in punctate structures within P6.p daughters. Indirect immunofluorescence of qaEx4003 [Ce-dab-1::Ce-DAB-1::GFP; unc-119(+)]; unc-119(e2498) animals with antibodies to LET-23 (left) and Ce-DAB$1:$ GFP (anti-GFP; center) and merged images (right) are shown. Images are single $0.2-\mu \mathrm{m}$ confocal planes through P6.p after one division.
Lipoprotein receptors interact with EGL-17

During synthesis and processing, receptor extracellular domains are in the lumen of the ER, Golgi, and transport vesicles, where they might associate directly or indirectly with EGL-17. Because the enormous extracellular domains of Ce-LRP-1 and Ce-LRP-2 ( 4600 and 4730 residues, respectively) were difficult to express, we first measured binding of EGL-17 to the extracellular domains of mammalian ApoER2 and VLDLR. Like Ce-
LRP-1 and Ce-LRP-2, these receptors contain blocks of complement-like LDLR type A repeats, EGF repeats, and six-bladed YWTD $\beta$ propellers (Fig. 6A). The extracellular domains of ApoER2 and VLDLR were expressed as fusions to the immunoglobulin Fc region (Hiesberger et al. 1999|, together with myc epitope-tagged EGL-17. The receptor-Fc fusion proteins were recovered by using Protein A and were tested for bound EGL-17-myc (Fig. 6B). Both ApoER2 and VLDLR associated with EGL-17-myc, 

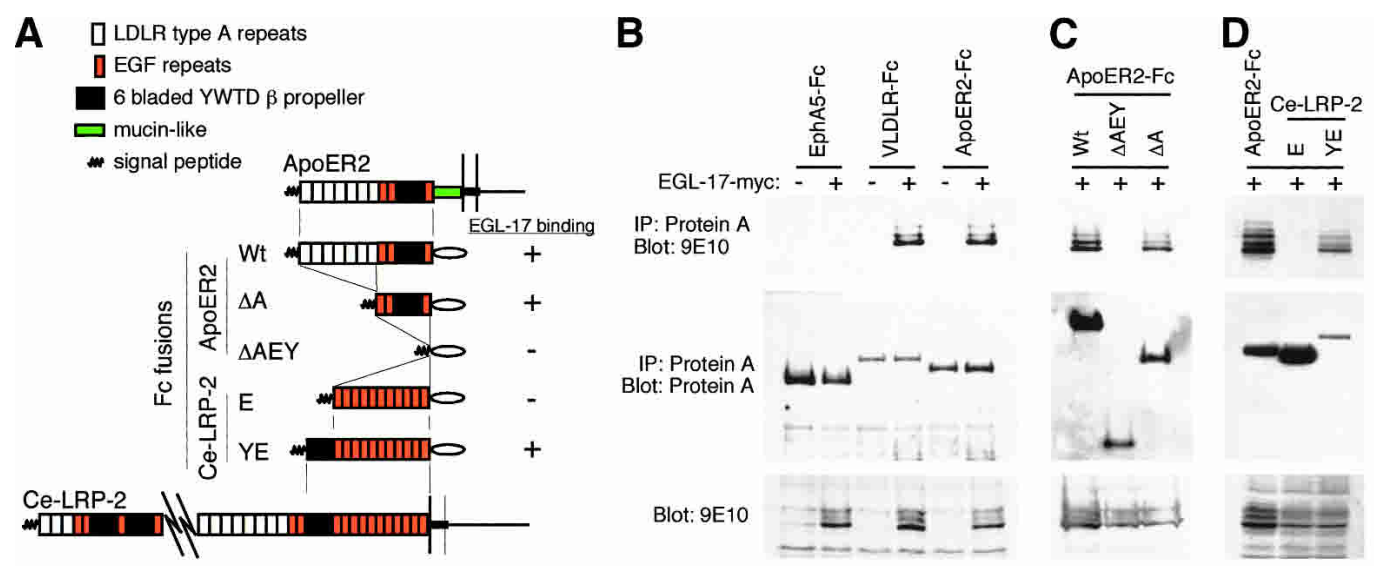

\section{$\mathbf{E}$}

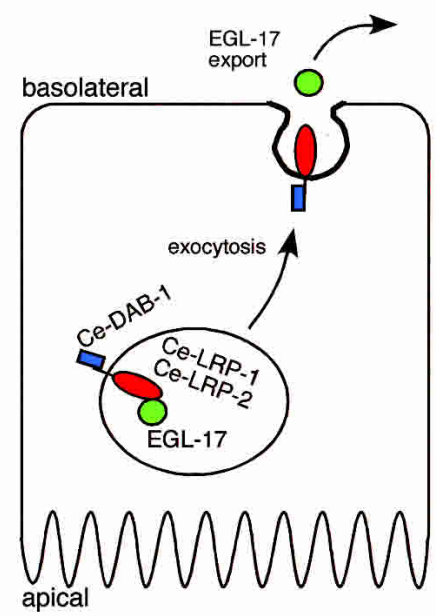

$\mathbf{F}$

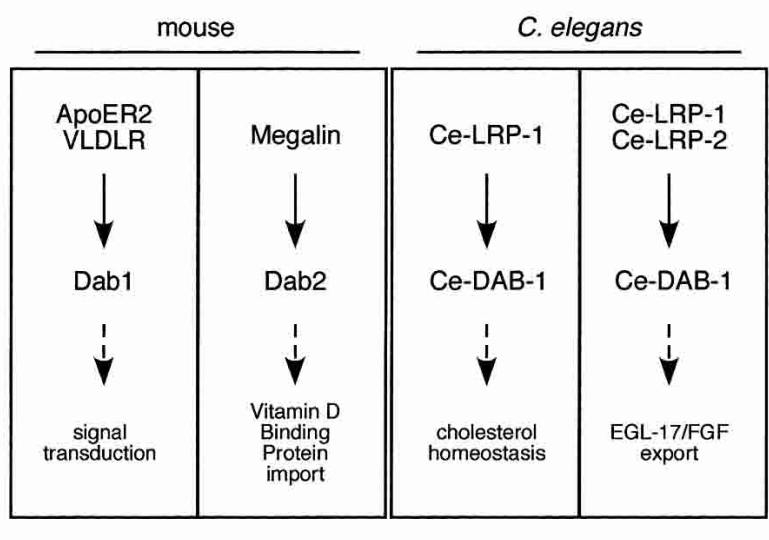

Figure 6. EGL-17 binds to the extracellular domains of lipoprotein receptors. $(A-D) 293 \mathrm{~T}$ cells were transfected with constructs encoding various extracellular domains fused to $\mathrm{Fc}$ in the presence or absence of EGL-17-myc. Lysates were precipitated with Protein A Sepharose, resolved by $9 \%$ SDS-PAGE, and analyzed by Western blotting. (A) Summary of constructs and results. (B) VLDLR and ApoER2, but not EphA5, coprecipitate EGL-17-myc (top). Expression levels of Fc fusion proteins (middle) and EGL-17-myc (bottom) are shown. $(C)$ The association of EGL-17 with ApoER2 is independent of the LDLR type A repeats. Expression levels of the ApoER2 $\triangle \mathrm{AEY}$ and ApoER2 $\Delta \mathrm{A}$ forms (middle) and EGL-17-myc (bottom) are shown. (D) EGL-17 binds to Ce-LRP-2 YWTD region. Despite weaker expression, Ce-LRP-2 YE coprecipitates EGL-17-myc, but Ce-LRP-2 E does not. $(E)$ Model. Ce-DAB-1 regulates the export of EGL-17 mediated through Ce-LRP-1 and Ce-LRP-2, possibly by direct interactions between Ce-DAB-1 in the cytosol, Ce-LRP-1 and Ce-LRP-2 in the membranes of secretory vesicles, and EGL-17 in the lumen of the vesicles. See text for discussion. $(F)$ Functions of lipoprotein receptors and Dab family members across species.

whereas the unrelated EphA5 did not (Fig. 6B). This suggests that EGL-17 can bind to the extracellular domains of mammalian lipoprotein receptors.

To identify the domains responsible for EGL-17 binding, we made deletions of the ApoER2-Fc fusion protein. Surprisingly, deletion of the LDLR type A repeats, which bind multiple ApoE- and ApoB-containing lipoproteins (Nykjaer and Willnow 2002), had no effect on EGL-17 binding (Fig. 6C; ApoER2 $\Delta$ A). However, further removal of three EGF repeats and the $\beta$ propeller abolished binding (ApoER2 $\triangle \mathrm{AEY}$ ). This suggests that the $\beta$ propeller and/or EGF repeats are required to bind to EGL-17.

To determine whether a C. elegans lipoprotein receptor can bind to EGL-17, we inserted various regions of Ce-LRP-2 into the minimal ApoER2-Fc $\triangle$ AEY construct. EGL-17-myc bound to a fusion containing the eighth membrane-proximal $\beta$ propeller and 11 EGF repeats from Ce-LRP-2 (Ce-LRP-2 YE), but not to a similar construct containing only the EGF repeats (Ce-LRP-2 E; Fig. 6D). This suggests that the association between Ce-LRP-2 and EGL-17 may be mediated by one of the $\beta$ propellers present in the extracellular region of Ce-LRP-2. This physical interaction may be important for the ability of Ce-LRP-2 to regulate EGL-17 transport in the developing vulva.

\section{Discussion}

We have found that two lipoprotein receptors, Ce-LRP-1 and Ce-LRP-2, and an adaptor protein, Ce-DAB-1, mediate secretion of EGL-17 from the C. elegans VPC P6.p and its descendants. Interfering with expression of either 
Ce-dab-1 or both Ce-lrp-1 and Ce-lrp-2 reduced SM migration, slowed egg laying, and caused accumulation of EGL-17::GFP in P6.p descendants (Figs. 1, 3, 4). A similar accumulation of EGL-17::GFP in P6.p descendants was observed by using RNAi for subunits of clathrin adaptor complex AP-1, but not AP-2 or AP-3. On the other hand, traffic of LET-23 to the apical surface of the VPCs, and its localization to the basolateral surface by the LIN-2/7/10 complex (Simske et al. 1996; Kaech et al. 1998; Whitfield et al. 1999), appears to be normal, and the protein AJM-1 (Koppen et al. 2001) also localizes normally to junctions (Fig. 3). Moreover, the LET-23 signal sequence, fused directly to either GFP or an EGL$17::$ GFP fusion protein, supports secretion that is independent of Ce-DAB-1 (data not shown). Thus, the effects of Ce-DAB-1 on EGL-17 traffic are specific and consistent with a requirement for secretion. The cytoplasmic tails of Ce-LRP-1 and Ce-LRP-2 can bind to the CeDAB-1 PTB domain (Fig. 5), whereas a conserved $\beta$ propeller domain in the extracellular regions of lipoprotein receptors can bind to EGL-17 (Fig. 6A-D), suggesting that protein-protein interactions link Ce-DAB-1 via the receptors to EGL-17. Ce-DAB-1 also localizes to Golgiproximal subcellular structures when expressed in tissue culture cells, and to vesicles in P6.p descendants. These results support a model shown in Figure 6E. In this model, Ce-DAB-1 is recruited to transport vesicles at some stage of the secretory pathway and aids in the sorting of Ce-LRP-1 and Ce-LRP-2 into nascent export vesicles. We propose that EGL-17 is cosorted via a direct or indirect interaction with the extracellular domains of the receptors (Fig. 6E). Ce-DAB-1 may also interact with other proteins important for vesicle budding or targeting.

Although it seems likely that reduced EGL-17 secretion from the developing vulva is a primary cause of the observed defects in SM migration and Egl, Ce-LRP-1, CeLRP-2, and Ce-DAB-1 may play additional roles. SM migration is regulated by a balance between gonad-dependent attraction and repulsion (GDA and GDR, respectively; Chen and Stern 1998). EGL-17 expression in the early gonad acts as the major attractant for the SMs (Branda and Stern 2000). The role of EGL-17 coming from the developing vulva is less clear, but is sufficient to precisely position the SMs in the absence of the gonad (Burdine et al. 1998). Although we detect some expression of Ce-DAB-1::GFP in the anchor cell at later stages of development (Fig. 2A), expression in the early gonad may be below our current limit of detection. Interestingly, Ce-dab-1 (RNAi) effects on SM migration are weaker than those described for null mutants of egl-17 (Stern and Horvitz 1991). This may reflect reduced, but not eliminated, EGL-17 export, disrupting the balance between attractive and repulsive cues. It is also possible that EGL-17 from the VPCs regulates SM function or differentiation. Thus, the Egl phenotype of Ce-dab-1 (RNAi) animals may reflect a reduction both in SM migration and in SM function.

In addition to a role in secretion, we also found that depletion of Ce-DAB-1 or Ce-LRP-1, but not Ce-LRP-2, causes cuticle Mlt deficiencies similar to those caused by cholesterol starvation or Ce-lrp-1 mutation (Yochem et al. 1999). This suggests that Ce-DAB-1 and Ce-LRP-1 have an additional role in cholesterol homeostasis. However, impaired cholesterol homeostasis due to Ce-lrp-1 RNAi is not sufficient to reduce SM migration. Interestingly, a genome-wide RNAi screen (Kamath et al. 2003) found that Ce-dab-1 (RNAi) animals are Unc, Gro, Slu, and Emb. Although we only observed the $\mathrm{F}_{\mathrm{O}}$ generation and thus did not observe any embryonic lethality (Emb), we did observe the other phenotypes. These phenotypes may be secondary to the cuticle Mlt deficiency, which could be missed in a large-scale screen or could reflect other cellular functions for Ce-DAB-1.

The model suggested by our results does not define the step in EGL-17 secretion that is facilitated by Ce-DAB-1 and the receptors. Each stage of vesicular traffic requires the coordination of coat protein assembly with the selection of target-specific receptors and the appropriate vesicle contents (Schmid 1997; Lippincott-Schwartz et al. 2000). Ce-LRP-1 and Ce-LRP-2 might function as cargo selection receptors and use Ce-DAB-1 to coordinate coat protein assembly at any of several steps. For example, cargo selection could occur at the ER to Golgi stage. Previously, coassembly of transmembrane proteins and COPII coats has been shown to allow concentrative sorting of receptor-associated soluble proteins in the ER lumen (Malkus et al. 2002). In yeast, Erv29p is a transmembrane protein that is responsible for sorting $\alpha$-factor to the Golgi (Malkus et al. 2002). In vertebrate cells, transmembrane proteins, including BAP31, ERGIC-53, and p24-family proteins, appear to select soluble proteins for transport from ER to Golgi (LippincottSchwartz et al. 2000). Similarly, if a Ce-DAB-1/receptor/ EGL-17 complex forms in the ER, then Ce-DAB-1 might link these components to COPII coat assembly to facilitate transport to the Golgi.

Alternatively, a Ce-DAB-1/receptor complex may be involved in traffic of EGL-17 from the trans-Golgi network (TGN) to the basolateral surface of P6.p and its descendants. This would be akin to the role of GGA proteins, which bind to a DXXL motif in the tail of the mannose 6-phosphate receptor and traffic it, and its associated soluble cargo, from the TGN to endosomes (Puertollano et al. 2001; Zhu et al. 2001). In mammalian epithelial cells, basolateral targeting of the LDL receptor depends on $\mu 1 \mathrm{~B}$-containing clathrin adaptor proteins and an FXNPXY signal, but it is not known whether $\mu 1 \mathrm{~B}$ binds to the signal directly or via another protein (Nelson and Yeaman 2001). The PTB-containing protein ARH was recently shown to be capable of binding to AP-1 components and to the FXNPXY of the LDL receptor, although it does not localize to the TGN (Mishra et al. 2002b). Our data suggest that Ce-DAB-1 may be involved in recruiting a clathrin adaptor to the tails of CeLRP-1 and Ce-LRP-2 at the trans-Golgi network in P6.p and its descendants in the C. elegans developing vulva.

A third possibility is that Ce-DAB-1 might be involved in binding of EGL-17 to recycling lipoprotein receptors in a common endosome, where ligands and receptors from the cell surface can associate and enter the secre- 
tory pathway without passing to lysosomes (Mostov et al. 2000). This hypothesis is consistent with the recent observations that lipoprotein receptors participate in transcytosis (Nelson and Yeaman 2001). Apical to basolateral transcytosis of LDL and thyroglobulin and vitamin D binding protein involves the LDLR and Megalin, respectively (Dehouck et al. 1997; Marino et al. 2000, 2001). During transcytosis, vesicles move between basolateral and apical common endosome compartments, where they may encounter newly synthesized proteins coming from the TGN (Mostov et al. 2000). In this way, endocytosed lipoprotein receptors that are either recycled or transcytosed may be able to carry newly synthesized ligands to their target membranes. Because CeDAB-1 binds to the FXNPXY endocytosis signal, it may assist delivery of Ce-LRP-1 and Ce-LRP-2 to the common endosome in order to collect EGL-17 for delivery to the surface, or may help target them from the common endosome to the basolateral surface. Distinguishing these possibilities will require detailed subcellular analysis of protein traffic in P6.p and its descendants.

\section{Evolutionarily conserved interactions between Disabled family proteins and lipoprotein receptors}

Dab family adaptor proteins interact functionally with lipoprotein receptors in both nematodes and mammals, even though the biological processes they mediate vary greatly (Fig. 6F). Ce-DAB-1 regulates secretion, Dab2 regulates endocytosis in the kidney (Mishra et al. 2002a; Morris et al. 2002), and Dab1 relays extracellular signals during brain development (Howell and Herz 2001), each via lipoprotein receptors. Although the role of Ce-DAB-1 in signaling is unclear, the high degree of functional conservation across species suggests that vertebrate Dab family members or other PTB-containing proteins may participate in regulated traffic of lipoprotein receptors and associated cargoes to the cell surface. Indeed, it is possible that an early embryonic requirement for Dab2 (Morris et al. 2002) might be a consequence of altered protein traffic in polarized epithelial cells of the embryo.

\section{Materials and methods}

Nematode culture and microscopy

Nematodes were cultured and manipulated at room temperature by using standard techniques. The standard wild-type strain is Bristol N2. Strains used were egl-17(n1377), unc119(e2498), Irp-1(ku156)/gld-1(q266), PD4666[ayIs6(X)] CeTwist hlh-8::GFP (Harfe et al. 1998), and ayIs4[egl-17::GFP; dpy-20(+)]; dpy-20(e1282ts) (Burdine et al. 1998). Strains from this work include the following: qaIs4000[Ce-dab-1::GFP; unc119(+)]; unc-119(e2498), qaEx4002[egl-17::EGL-17::GFP; unc119(+)]; unc-119(e2498), qaEx4003[Ce-dab-1::Ce-DAB-1::GFP; unc-119(+)]; unc-119(e2498), qaIs4004[egl-17::EGL-17::GFP; unc-119(+)]; unc-119(e2498). For microscopy, worms were mounted on a $3 \%$ agarose pad in $\mathrm{M} 9\left(42 \mathrm{mM} \mathrm{Na}_{2} \mathrm{HPO}_{4}, 22 \mathrm{mM}\right.$ $\mathrm{KH}_{2} \mathrm{PO}_{4}, 85 \mathrm{mM} \mathrm{NaCl}, 1 \mathrm{mM} \mathrm{MgSO}_{4}$ ) supplemented with 10 $\mathrm{mM} \mathrm{NaN}_{3}$. All fluorescence and DIC /differential interference contrast) images were obtained by using a standard epi- fluorescence microscope equipped with a digital camera and processed with Adobe Photoshop 5.0 (Adobe Systems). For comparisons of image intensity, all images in a set were processed equivalently. Cells were identified on the basis of their positions as described (Sulston and Horvitz 1977; Sternberg and Horvitz 1986). When visible in the plane of focus shown, the AC is indicated by an arrowhead. Anterior is to the left; dorsal, to the top.

\section{Plasmids and injections}

Specifics of plasmid construction are available upon request. Transgenic animals were generated by using standard injection techniques (Fire 1986; Mello et al. 1991). Multiple independent strains carrying each transgene were screened for expression and phenotype. Representative strains were chosen for each experiment. All PCRs for cloning were performed by using Pfu Turbo (Stratagene), and open reading frames were sequenced to confirm polymerase fidelity. Expression plasmids for ApoER2-V5Fc, VLDLR-V5-Fc (Hiesberger et al. 1999), and EphA5-Fc (Davis et al. 1994) fusion proteins were constructed as described.

\section{dsRNA-mediated interference (RNAi) and phenotype scoring}

Standard techniques were used to synthesize dsRNA from T7 promoter-tagged, PCR-amplified cDNA. Templates for the synthesis of dsRNA are as follows: pDKF8 (Ce-dab-1), yk358b4 (1rp-1), yk8d2 (rme-2), yk597d6 (Ce-lrp-2), and $\mathrm{BSKS}^{+}$(control). After synthesis, RNA was ethanol-precipitated and resuspended in distilled $\mathrm{H}_{2} \mathrm{O}$. Eggs were collected from hypochlorite-treated gravid adults (Lewis and Fleming 1995), and after hatching, L1 larvae were soaked overnight at room temperature in $5 \mu \mathrm{L}$ of solution comprised of equivalent amounts of each RNA strand in $3 \mathrm{mM}$ spermidine. Soaked animals were then transferred to NGM plates seeded with E. coli OP-50 and allowed to develop until L2 to L4, when they were observed and photographed as described above. When multiple RNAi species were used, equivalent amounts of each RNA strand were used. Egl rate experiments were performed as described (Byerly et al. 1976). For scoring EGL-17::GFP retention, photographic exposures were set to Ce-dab-1 (RNAi) animals. Equal exposure times were then used to measure retention in controls and other RNAi treated animals.

\section{SM migration and egl-17(n1377) rescue}

SM studies were performed in the stain ayIs6(X) [CeTwist hlh$8:: G F P]$ (PD4666), which expresses GFP in the nucleus of the descendants of $M$, and specifically in the SMs and their descendants during L3 to L4 (Harfe et al. 1998). The final positions of the SMs were scored relative to the Pn.p cells as described (Tho mas et al. 1990). For egl-17(n1377) rescue, pDKF47[egl-17::EGL$17:: G F P](10 \mathrm{ng} / \mathrm{\mu L})$ or pDKF58[eg1-17::LET-23sp ::EGL-17::GFP] with $\mathrm{BSKS}^{+}(130 \mathrm{ng} / \mu \mathrm{L})$ was injected as above. To ensure hypodermal expression (from which the vulva is derived), pRF4[rol6(su1006)] (10 ng/ $\mu \mathrm{L}$; Mello et al. 1991) was used as an injection marker. $\mathrm{F}_{1}$ animals expressing the rol-6(su1006) marker were picked to individual NGM + OP-50 plates and were observed at least once a day for $4 \mathrm{~d}$ once becoming adults. If worms developed a "bag of worms" phenotype within $2 \mathrm{~d}$ of becoming adults, they were scored as positive for the phenotype. All other animals were scored as negative.

\section{Yeast two-hybrid interactions}

The physical interactions of lipoprotein receptor intracellular domains and the Ce-DAB-1 PTB domain were tested as de- 
scribed (Vojtek et al. 1997) by using plasmids pDKF40[VP-16 Ce-LRP-1], pDKF42[pLex10 Ce-DAB-1 PTB], and pDKF52[VP-16 Ce-LRP-2].

\section{Statistical analysis}

The significance of difference between distributions of the SMs were determined using a two-tailed Mann-Whitney $U$ test. For these tests, the position of each hash mark in the figures was converted to a number based on the distance of each SM away from P8p. For egl-17(n1377) rescue experiments, the significance of controls versus animals injected with pDKF47[ egl$17:: E G L-17:: G F P]$ DNA was determined by using the $\chi^{2}$-test.

\section{Immunoprecipitations}

Cells were maintained in DMEM $+10 \%$ FBS. One hundred-millimeter dishes of $293 \mathrm{~T}$ cells were transfected by using calcium phosphate with various combinations of ApoER2-V5-Fc, EphA5-Fc, pDKF33[eg1-17-myc], pDKF64[ApoER2 $\Delta$ AEY], pDKF65[ApoER2 $\Delta \mathrm{A}$ ], pDKF66[Ce-LRP-2 E], or pDKF67[CeLRP-2 YE] (6 $\mu \mathrm{g}$ each). The following day, the medium was replaced with DMEM $+10 \%$ FBS. Two days after transfection, the cells were rinsed one time in ice-cold PBS and lysed in 500 $\mu \mathrm{L}$ of lysis buffer ( $1 \%$ Triton X-100, $50 \mathrm{mM}$ Tris at $\mathrm{pH} 7.4,120$ $\mathrm{mM} \mathrm{NaCl}, 2 \mathrm{mM} \mathrm{CaCl}_{2}$ ). After clarification by centrifugation at 15,000 RPM, supernatants were precipitated with Protein A Sepharose (Sigma). Precipitated proteins or $20 \mu \mathrm{L}$ of cell lysate were resolved by $9 \%$ SDS-PAGE and transferred to nitrocellulose, and Western blots were performed by using 9E10 (antimyc) or Protein A-HRP (to detect Fc).

\section{Immunofluorescence}

LET-23 and AJM-1 were localized as described (Whitfield et al. 1999). Fixed animals were also stained with anti-GFP antibodies (mAb3E6, Q-Biogene) at 1:50. Confocal z-series were used to reconstruct projected three-dimensional images. For cell culture experiments, NIH3T3 or HeLa cells were transfected with calcium phosphate or Lipofectamine Plus (Invitrogen), respectively, on glass coverslips. Forty-eight hours later, cells were rinsed twice in PBS, fixed in $3.7 \%$ formaldehyde for $15 \mathrm{~min}$ at room temperature, and permeabilized with $0.1 \%$ Triton X-100 in PBS. Cells were stained with anti-Ce-DAB-1 monoclonal antibody P1F4-E5 culture supernatant (1:10), Texas Red wheat germ agglutinin (WGA; $12.5 \mu \mathrm{g} / \mathrm{mL}$, Molecular Probes), and anti- $\gamma$-adaptin (mAb 100/3, Sigma, 1:50). Isotype-specific secondary antibodies were used where appropriate.

\section{Acknowledgments}

We thank Barbara Page and Jeremy Nance for helpful discussions and experimental assistance. Tara Herrick performed the RNAi experiments for clathrin adaptor subunits. We thank Frank Gertler, Brian Howell, and Gian Garriga for their insights, as well as Katie Mickey for early experiments. We also thank the CGC, Michael Stern, the Priess Laboratory, and Yuji Kohara for worm strains and ESTs; Joachim Herz, Stuart Kim, Jeff Simske, and Jason Pitt for reagents and methods; and Priscilla Kronstad-O'Brien for technical assistance. We thank Jeremy Nance, Jim Priess, Leslie Cary, and Miriam Rosenberg for the critical reading of a draft of this manuscript. D.M.K. was supported by a Terry Fox Fellowship with funds from the Canadian Cancer Society and the National Cancer Institute of Canada.
This work was supported in part by National Institutes of Health grants CA41072 and GM066257.

The publication costs of this article were defrayed in part by payment of page charges. This article must therefore be hereby marked "advertisement" in accordance with 18 USC section 1734 solely to indicate this fact.

\section{References}

Amanai, K. and Jiang, J. 2001. Distinct roles of Central missing and Dispatched in sending the Hedgehog signal. Development 128: 5119-5127.

Borland, C.Z., Schutzman, J.L., and Stern, M.J. 2001. Fibroblast growth factor signaling in Caenorhabditis elegans. BioEssays 23: 1120-1130.

Branda, C.S. and Stern, M.J. 2000. Mechanisms controlling sex myoblast migration in Caenorhabditis elegans hermaphrodites. Dev. Biol. 226: 137-151.

Burdine, R.D., Chen, E.B., Kwok, S.F., and Stern, M.J. 1997. egl-17 encodes an invertebrate fibroblast growth factor family member required specifically for sex myoblast migration in Caenorhabditis elegans. Proc. Natl. Acad. Sci. 94: 24332437.

Burdine, R.D., Branda, C.S., and Stern, M.J. 1998. EGL-17(FGF) expression coordinates the attraction of the migrating sex myoblasts with vulval induction in C. elegans. Development 125: 1083-1093.

Byerly, L., Cassada, R.C., and Russell, R.L. 1976. The life cycle of the nematode Caenorhabditis elegans, I: Wild-type growth and reproduction. Dev. Biol. 51: 23-33.

Chen, E.B. and Stern, M.J. 1998. Understanding cell migration guidance: Lessons from sex myoblast migration in C. elegans. Trends Genet. 14: 322-327.

Christensen, E.I. and Birn, H. 2002. Megalin and Cubulin: Multifunctional endocytic receptors. Nat. Rev. Mol. Cell. Biol. 3: $258-268$.

Davis, S., Gale, N.W., Aldrich, T.H., Maisonpierre, P.C., Lhotak, V., Pawson, T., Goldfarb, M., and Yancopoulos, G.D. 1994. Ligands for EPH-related receptor tyrosine kinases that require membrane attachment or clustering for activity. Science 266: 816-819.

Dehouck, B., Fenart, L., Dehouck, M.P., Pierce, A., Torpier, G., and Cecchelli, R. 1997. A new function for the LDL receptor: Transcytosis of LDL across the blood-brain barrier. J. Cell Biol. 138: 877-889.

DeVore, D.L., Horvitz, H.R., and Stern, M.J. 1995. An FGF receptor signaling pathway is required for the normal cell migrations of the sex myoblasts in C. elegans hermaphrodites. Cell 83: 611-620.

Fire, A. 1986. Integrative transformation of Caenorhabditis elegans. EMBO J. 5: 2673-2680.

Fire, A., Xu, S., Montgomery, M.K., Kostas, S.A., Driver, S.E., and Mello, C.C. 1998. Potent and specific genetic interference by double-stranded RNA in Caenorhabditis elegans. Nature 391: 806-811.

Folsch, H., Ohno, H., Bonifacino, J.S., and Mellman, I. 1999. A novel clathrin adaptor complex mediates basolateral targeting in polarized epithelial cells. Cell 99: 189-198.

Friesel, R.E. and Maciag, T. 1995. Molecular mechanisms of angiogenesis: Fibroblast growth factor signal transduction. FASEB J. 9: 919-925.

Goldfarb, M. 1996. Functions of fibroblast growth factors in vertebrate development. Cytokine Growth Factor Rev. 7: 311-325

Grant, B. and Hirsh, D. 1999. Receptor-mediated endocytosis in 
the Caenorhabditis elegans oocyte. Mol. Biol. Cell 10: 43114326.

Harfe, B.D., Vaz Gomes, A., Kenyon, C., Liu, J., Krause, M., and Fire, A. 1998. Analysis of a Caenorhabditis elegans Twist homolog identifies conserved and divergent aspects of mesodermal patterning. Genes \& Dev. 12: 2623-2635.

Herz, J. and Strickland, D.K. 2001. LRP: A multifunctional scavenger and signaling receptor. J. Clin. Invest. 108: 779-784.

Hiesberger, T., Trommsdorff, M., Howell, B.W., Goffinet, A., Mumby, M.C., Cooper, J.A., and Herz, J. 1999. Direct binding of Reelin to VLDL receptor and ApoE receptor 2 induces tyrosine phosphorylation of disabled-1 and modulates $\tau$ phosphorylation. Neuron 24: 481-489.

Howell, B.W. and Herz, J. 2001. The LDL receptor gene family: Signaling functions during development. Curr. Opin. Neurobiol. 11: 74-81.

Howell, B.W., Lanier, L.M., Frank, R., Gertler, F.B., and Cooper, J.A. 1999. The disabled 1 phosphotyrosine-binding domain binds to the internalization signals of transmembrane glycoproteins and to phospholipids. Mol. Cell. Biol. 19: 51795188.

Kaech, S.M., Whitfield, C.W., and Kim, S.K. 1998. The LIN-2/ LIN-7/LIN-10 complex mediates basolateral membrane localization of the C. elegans EGF receptor LET-23 in vulval epithelial cells. Cell 94: 761-771.

Kamath, R.S., Fraser, A.G., Dong, Y., Poulin, G., Durbin, R., Gotta, M., Kanapin, A., Le Bot, N., Moreno, S., Sohrmann, M., et al. 2003. Systematic functional analysis of the Caenorhabditis elegans genome using RNAi. Nature 421: 231237.

Koppen, M., Simske, J.S., Sims, P.A., Firestein, B.L., Hall, D.H., Radice, A.D., Rongo, C., and Hardin, J.D. 2001. Cooperative regulation of AJM-1 controls junctional integrity in Caenorhabditis elegans epithelia. Nat. Cell. Biol. 3: 983-991.

Lee, J.R., Urban, S., Garvey, C.F., and Freeman, M. 2001. Regulated intracellular ligand transport and proteolysis control EGF signal activation in Drosophila. Cell 107: 161-171.

Lewis, J.A. and Fleming, J.T. 1995. Basic culture methods. In Caenorhabditis elegans: Modern biological analysis of an organism (eds. H.F. Epstein and D.C. Shakes), pp. 3-29. Academic Press, San Diego.

Lippincott-Schwartz, J., Roberts, T.H., and Hirschberg, K. 2000. Secretory protein trafficking and organelle dynamics in living cells. Ann. Rev. Cell Dev. Biol. 16: 557-589.

Maeda, I., Kohara, Y., Yamamoto, M., and Sugimoto, A. 2001. Large-scale analysis of gene function in Caenorhabditis elegans by high-throughput RNAi. Curr. Biol. 11: 171-176.

Malkus, P., Jiang, F., and Schekman, R. 2002. Concentrative sorting of secretory cargo proteins into COPII-coated vesicles. J. Cell Biol. 159: 915-921.

Marino, M., Zheng, G., Chiovato, L., Pinchera, A., Brown, D., Andrews, D., and McCluskey, R.T. 2000. Role of megalin (gp330) in transcytosis of thyroglobulin by thyroid cells: A novel function in the control of thyroid hormone release. $J$. Biol. Chem. 275: 7125-7137.

Marino, M., Andrews, D., Brown, D., and McCluskey, R.T. 2001. Transcytosis of retinol-binding protein across renal proximal tubule cells after megalin (gp 330)-mediated endocytosis. J. Am. Soc. Nephrol. 12: 637-648.

Mello, C.C., Kramer, J.M., Stinchcomb, D., and Ambros, V. 1991. Efficient gene transfer in C. elegans: Extrachromosomal maintenance and integration of transforming sequences. EMBO J. 10: 3959-3970.

Mishra, S.K., Keyel, P.A., Hawryluk, M.J., Agostinelli, N.R., Watkins, S.C., and Traub, L.M. 2002a. Disabled-2 exhibits the properties of a cargo-selective endocytic clathrin adap- tor. EMBO J. 21: 4915-4926.

Mishra, S.K., Watkins, S.C., and Traub, L.M. 2002b. The autosomal recessive hypercholesterolemia (ARH) protein interfaces directly with the clathrin-coat machinery. Proc. Natl. Acad. Sci. 99: 16099-16104.

Morris, S.M. and Cooper, J.A. 2001. Disabled-2 colocalizes with the LDLR in clathrin-coated pits and interacts with AP-2. Traffic 2: 111-123.

Morris, S.M., Tallquist, M.D., Rock, C.O., and Cooper, J.A. 2002. Dual roles for the Dab2 adaptor protein in embryonic development and kidney transport. EMBO J. 21: 1555-1564.

Mostov, K.E., Verges, M., and Altschuler, Y. 2000. Membrane traffic in polarized epithelial cells. Curr. Opin. Cell Biol. 12: $483-490$.

Nelson, W.J. and Yeaman, C. 2001. Protein trafficking in the exocytic pathway of polarized epithelial cells. Trends Cell Biol. 11: 483-486.

Newman, A.P., White, J.G., and Sternberg, P.W. 1996. Morphogenesis of the C. elegans hermaphrodite uterus. Development 122: 3617-3626.

Nykjaer, A. and Willnow, T.E. 2002. The low-density lipoprotein receptor gene family: A cellular Swiss army knife? Trends Cell Biol. 12: 273-280.

Ornitz, D.M. and Itoh, N. 2001. Fibroblast growth factors. Genome Biol. 2: reviews3005.1-reviews3005.9.

Puertollano, R., Aguilar, R.C., Gorshkova, I., Crouch, R.J., and Bonifacino, J.S. 2001. Sorting of mannose 6-phosphate receptors mediated by the GGAs. Science 292: 1712-1716.

Schmid, S.L. 1997. Clathrin-coated vesicle formation and protein sorting: An integrated process. Annu. Rev. Biochem. 66: 511-548.

Shim, J., Sternberg, P.W., and Lee, J. 2000. Distinct and redundant functions of mul medium chains of the AP-1 clathrinassociated protein complex in the nematode Caenorhabditis elegans. Mol. Biol. Cell 11: 2743-2756.

Shin-i, T. and Kohara, Y. 2001. The Nematode Expression Pattern DataBase. http://nematode.lab.nig.ac.jp/db/readme. html.

Simske, J.S., Kaech, S.M., Harp, S.A., and Kim, S.K. 1996. LET23 receptor localization by the cell junction protein LIN-7 during C. elegans vulval induction. Cell 85: 195-204.

Springer, T.A. 1998. An extracellular $\beta$-propeller module predicted in lipoprotein and scavenger receptors, tyrosine kinases, epidermal growth factor precursor, and extracellular matrix components. J. Mol. Biol. 283: 837-862.

Stern, M.J. and Horvitz, H.R. 1991. A normally attractive cell interaction is repulsive in two C. elegans mesodermal cell migration mutants. Development 113: 797-803.

Sternberg, P.W. and Horvitz, H.R. 1986. Pattern formation during vulval development in C. elegans. Cell 44: 761-772.

Sulston, J.E. and Horvitz, H.R. 1977. Post-embryonic cell lineages of the nematode, Caenorhabditis elegans. Dev. Biol. 56: 110-156.

Sutherland, D., Samakovlis, C., and Krasnow, M.A. 1996. branchless encodes a Drosophila FGF homolog that controls tracheal cell migration and the pattern of branching. Cell 87: 1091-1101.

Tabara, H., Grishok, A., and Mello, C.C. 1998. RNAi in C. elegans: Soaking in the genome sequence. Science 282: 430431.

Tanaka, K., Kitagawa, Y., and Kadowaki, T. 2002. Drosophila segment polarity gene product porcupine stimulates the posttranslational N-glycosylation of wingless in the endoplasmic reticulum. J. Biol. Chem. 277: 12816-12823.

Thomas, J.H., Stern, M.J., and Horvitz, H.R. 1990. Cell interactions coordinate the development of the C. elegans egg-lay- 
ing system. Cell 62: 1041-1052.

Trommsdorff, M., Gotthardt, M., Hiesberger, T., Shelton, J., Stockinger, W., Nimpf, J., Hammer, R.E., Richardson, J.A., and Herz, J. 1999. Reeler/Disabled-like disruption of neuronal migration in knockout mice lacking the VLDL receptor and ApoE receptor 2. Cell 97: 689-701.

van den Heuvel, M., Harryman-Samos, C., Klingensmith, J., Perrimon, N., and Nusse, R. 1993. Mutations in the segment polarity genes wingless and porcupine impair secretion of the wingless protein. EMBO J. 12: 5293-5302.

Vojtek, A.J., Cooper, J.A., and Hollenberg, S.M. 1997. Searching for interacting proteins with the two-hybrid system II. In The yeast two-hybrid system (eds. P.L. Bartel and S. Fields), pp. 29-42. Oxford University Press, New York.

Whitfield, C.W., Benard, C., Barnes, T., Hekimi, S., and Kim, S.K. 1999. Basolateral localization of the Caenorhabditis elegans epidermal growth factor receptor in epithelial cells by the PDZ protein LIN-10. Mol. Biol. Cell 10: 2087-2100.

Yochem, J., Tuck, S., Greenwald, I., and Han, M. 1999. A gp330/ megalin-related protein is required in the major epidermis of Caenorhabditis elegans for completion of molting. Development 126: 597-606.

Zhu, Y., Doray, B., Poussu, A., Lehto, V.P., and Kornfeld, S. 2001. Binding of GGA2 to the lysosomal enzyme sorting motif of the mannose 6-phosphate receptor. Science 292: $1716-1718$. 


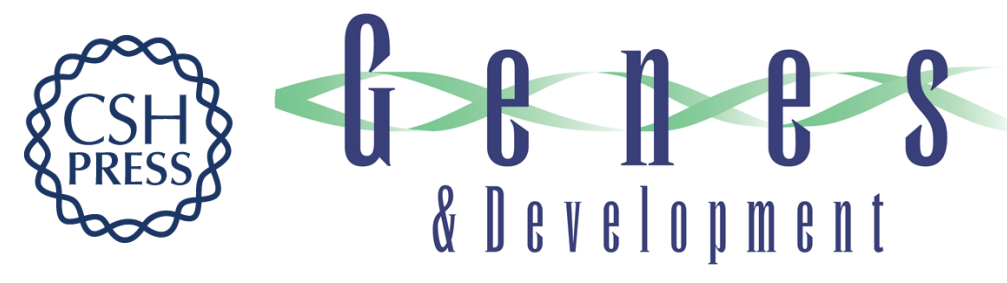

\section{Lipoprotein receptors and a Disabled family cytoplasmic adaptor protein regulate EGL-17/FGF export in C. elegans}

Darren M. Kamikura and Jonathan A. Cooper

Genes Dev. 2003, 17:

Access the most recent version at doi:10.1101/gad.1136103

References This article cites 57 articles, 22 of which can be accessed free at: http://genesdev.cshlp.org/content/17/22/2798.full.html\#ref-list-1

License

Email Alerting

Receive free email alerts when new articles cite this article - sign up in the box at the top Service right corner of the article or click here.

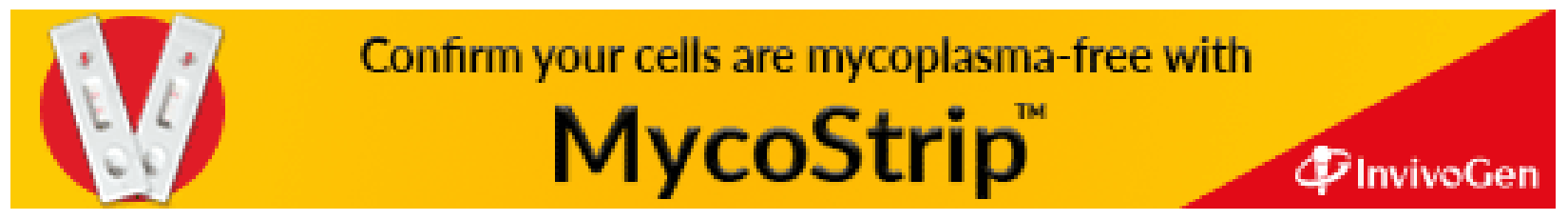

